\title{
Variation in stratum corneum protein content as a function of anatomical site and ethnic group
}

N Raj, R Voegeli\#, AV Rawlings, S Gibbons, MR Munday, B Summers§ and ME Lane UCL School of Pharmacy, London, UK, \#DSM Nutritional Products Ltd., Kaiseraugst, Switzerland, § Photobiology Laboratory, Sefako Makgatho University, South Africa

\section{OBJECTIVES}

Quantification of stratum corneum (SC) protein levels from tape strippings is frequently used to investigate skin conditions, to correct for amounts of SC protein removed in SC biomarker studies and to determine distribution of topically applied ingredients. In recent years, a rapid and convenient method for SC protein quantification from tape strippings has become available using infrared densitometry (IRD). However, standard curves have only been generated for Caucasian forearm and shoulder SC and have been assumed to be correct not only for facial SC but also for SC samples of other ethnic groups. The aim of the present study was to investigate whether the use of IRD for SC protein measurement is valid for other body sites such as the cheek and for measuring SC protein content of darkly pigmented skin types.

\section{METHODS}

Ten Caucasian and ten Black African female subjects with self-assessed normal skin participated in the study. Tape strippings were collected from two different body sites (forearm and cheek). First from the tape strippings the SC optical absorption was determined densitometrically. This obtained absorption (\%) was compared with absolute SC protein extracted from the same tapes using a colorimetric micro bicinchoninic acid ( $\mu \mathrm{BCA})$ assay. 


\section{RESULTS}

Higher amounts of SC protein were removed from the forearm compared with the cheek $(p<0.01)$. The absolute SC protein concentration quantified by $\mu B C A$ assay and the absorption of SC proteins by IRD followed a similar profile. There was no significant difference found between the two ethnicities in SC protein $(p>0.05)$. The overall coefficient of determination $\left(R^{2}\right)$ shows a good fit to the regression line between the two methods in both sites (forearm $=0.82$, cheek $=0.77$ ). Also, both ethnicities showed good correlation $\left(R^{2} \geq 0.69, p=0.01\right)$.

\section{CONCLUSIONS}

Facial SC is morphologically distinct from the forearm, as demonstrated by the differences in amounts of SC removed. Although the data distribution in different subject groups varied, the regression was always quite similar between the two body sites and both ethnic groups. Also the correlations were similar to previously published data on other body sites. The resultant calibration curves can be used as a rapid indirect protein assessment of tape strippings from the cheek.

\section{Key words}

Infrared densitometry, tape stripping, stratum corneum protein, skin ethnicities, SC cohesion, skin barrier 


\section{Introduction}

The stratum corneum (SC) consists of corneodesmosome-bound corneocytes derived from differentiated keratinocytes tightly stacked within lipid bilayers. This well-evolved barrier provides protection against external physical challenges and prevents excessive transepidermal water loss (TEWL) [1]. An understanding of this natural barrier, which supports life in a terrestrial environment, is necessary in order to develop better formulations that can target or permeate to deeper skin layers. The thickness of the SC, its protein content and TEWL measurements have previously been used as indices of SC integrity [2-8]. The minimally invasive method of tape stripping has been used extensively for determining not only the endogenous and exogenous molecules within the SC but also the SC thickness and protein content. This involves removal of sequential layers of the SC using adhesive tapes and can be performed with minimum discomfort to participants and relative ease in vivo. It has become a standard technique in dermatological, pharmaceutical and cosmetic research since its introduction by Pinkus in 1951 [9-12]. However, the amount of SC removed by tape stripping is not linearly proportional to the number of tapes removed [12-14]. Tape stripping works on the principle of adhesiveness; hence as the cohesive force between corneocytes increases, the amount of skin removed by tape stripping decreases [15]. Oily formulations can influence the adhesiveness of SC to the tapes [16]. Therefore the number of tape strippings removed is not a valid method to characterize the penetration of any topically applied formulations [12]. Another factor, which influences the quantity of SC removed, is the thickness of the SC. This can vary with each individual and with anatomic site [17]. The force applied together with the water content of SC also influences the number of cells removed by 
tape stripping. The interval time between application and removal of tapes has also been reported to have a profound effect on SC recovery [17]. Finally, the manner of removing the tapes from skin $[14,18]$ and occlusion of the SC also influence the quantity of SC harvested using tapes [17].

There are various methods currently available to quantify SC removed on tape strippings. The simplest approach is based on weight differences between the tape strippings. This method is labour intensive and time consuming and subject to artifacts [14]. Another is based on ultraviolet (UV) spectrophotometry that utilizes the absorption maximum of proteins at $278 \mathrm{~nm}$ [19]. However, overlapping layers of SC on a single tape stripping causes light scattering, which affects the consistency of results [14]. A third is a colorimetric method based on a commercially available protein assay kit that was initially reported by Dreher and co-workers [20]. This method was further modified to reduce time of sample measurement by the use of a 96 well microplate method [21]. The major disadvantage of this measurement is the necessity for destruction of individual tape strippings. A fourth method utilizing UV and visible range $(430 \mathrm{~nm})$ absorbance, introduced the major advantage nondestructive tape analysis [22]. However, interference from other chemicals with similar absorption wavelengths as corneocyte proteins may compromise the accuracy of these methods. Quantification of covering density of SC on tapes using classic microscopy and laser scanning microscopy has also been investigated, but is associated with long processing times [23].

More recently Voegeli et al. have reported an absorbance based quantification method for human SC samples utilizing the $850 \mathrm{~nm}$ infrared region (IR) [24]. The peak wavelength of $850 \mathrm{~nm}$ not only prevents any thermal denaturation of 
biomolecules but also reduces the loss of light by scattering [24]. Spectroscopic absorption is used indirectly to quantify the protein density on a specific area of tape [24]. The absorption values (\%) can be read directly from the display, which makes the method rapid and non-destructive. Linear correlations between the optical pseudo-absorption and respective SC protein content have been reported for in vivo and in vitro studies [24-26]. This method has also been shown to correlate with the gravimetric approach [27]. The amount of protein present in the SC has also been used to assess conditions such as skin dryness [30]. However, the regression of infrared densitometry (IRD) and SC protein was previously determined using SC samples from the forearm and shoulder (male) and was assumed to be similar for other body sites. Moreover, studies were only conducted using Caucasian samples and there is a need to confirm the relevance of those regressions with samples from other ethnicities. Previously the number of SC cell layers has been shown to differ for Caucasian and African American subjects [28]. An objective of the present work was therefore to determine any variation in protein determination when using IRD to profile SC samples from Black African female subjects.

Equally, the validity of IRD compared with micro bicinchoninic acid $(\mu B C A)$ to profile facial SC areas in human subjects has not been investigated to date. The $\mu \mathrm{BCA}$ method relies on the ability of proteins to catalyze the reduction of $\mathrm{Cu} 2+$ to $\mathrm{Cu}+$, which then forms a colored complex with bicinchonic acid. This method is highly sensitive but the destructive nature of assay makes it less suitable.

The face is one of the most exposed skin sites of the human body and is subject to considerable environmental stress, e.g. temperature and humidity fluctuations. In 
addition, compared with other body sites facial skin shows elevated TEWL, increased SC cohesion and higher proteolytic activity resulting in premature corneodesmosomal degradation and SC thinning [29]. Also, the facial SC comprises of relatively smaller and immature corneocytes with a rapid turnover rate compared with other anatomical sites [30, 31]. The thickness of facial SC is approximately 16.8 $\mu \mathrm{m}$ whereas that of the forearm is $22.6 \mu \mathrm{m}$. In addition, there is considerable difference in the number of cell layers in SC between the two sites (7-9 layers) [32, 33]. This indicates that facial SC possesses a diminished stratum disjunctum (upper layers) and the remaining stratum compactum (lower layer) is responsible for increased SC cohesion [34]. Clearly, facial skin is structurally different from forearm skin and the validity of using the IRD approach to measure SC protein levels on the face needs to be evaluated [35, 36].

\section{Materials and Methods}

\section{Materials}

Sodium hydroxide (Lot. S/4920/53, analytical specification), hydrochloric acid $1 \mathrm{M}$ (J/4320/15, analytical specification), sodium chloride (S/3160/65, analytical specification) was procured from Fisher Scientific, Leicestershire, UK). The $\mu \mathrm{BCA}$ TM Protein Assay Kit was obtained from Pierce Biotechnology Inc., Rockford, USA. The kit consisted of reagent A (alkaline tartrate-carbonate buffer), reagent $B$ (bicinchoninic acid solution) and reagent $C$ (copper sulphate solution), which were mixed according to the manufacturer's instructions. The protein standards employed were bovine gamma globulin (BGG; $2 \mathrm{mg} / \mathrm{ml}$, product No. 23212,) as purchased from Pierce Biotechnology Inc., Rockford, USA. The microplates were measured 
using a multichannel absorbance microplate reader (BioTek ${ }^{\mathrm{TM}}$ ELx808). Standard DSquame ${ }^{\circledR}$ adhesive tapes with a diameter of $2.2 \mathrm{~cm}$ and an area of $3.8 \mathrm{~cm}^{2}$, a DSquame ${ }^{\circledR}$ pressure applicator and D-Squame ${ }^{\circledR}$ disc racks were obtained from Cuderm Corporation (Dallas, U.S.A). An orbital incubator SI50 (Stuart Scientific, Staffordshire, UK) was used for protein extraction from tapes. Absorption measurements at $850 \mathrm{~nm}$ were recorded with an infrared densitometer model SquameScan ${ }^{\mathrm{TM}}$ 850A (Heiland electronic, Wetzlar, Germany).

\section{Study Population and Study Design}

The study was a prospective cross-sectional study and was approved by the School of Health Care Sciences Research and Ethics committee (SREC) together with the Medunsa Campus Research and Ethics Committee (MREC) (Pretoria, South Africa) and was conducted in accordance with the Declaration of Helsinki Principles. Written, informed consent was obtained from all participants before enrolment. Ten female Caucasian (aged $44.6 \pm 3.1 \mathrm{yrs}$ ) and ten female Black African subjects (aged $38.2 \pm 2.3 \mathrm{yrs}$ ) all living in Pretoria, South Africa, with self-assessed normal skin participated in the study. Participants were required not to apply any dermatological or cosmetic products for at least $72 \mathrm{~h}$ before the SC was sampled. All the participants were acclimatised in a room under standard conditions at a temperature of $21 \pm 1{ }^{\circ} \mathrm{C}$ and a relative humidity of $35 \pm 10 \%$ for at least 30 min before tape stripping.

The skin was gently cleaned by swabbing with a cotton pad soaked in distilled water at ambient temperature and allowed to dry at room temperature. The skin sites were marked with a surgical marker to ensure that the tapes were consistently applied to the same area. Two different body sites (mid volar forearm and cheek, $3 \mathrm{~cm}$ vertically beneath the outer edge of the eye) were sampled by tape stripping. The 
tape strippings and IRD measurements were performed by the same technician to minimise personnel errors [17, 37].

\section{Tape Stripping}

Nine consecutive standard D-Squame ${ }^{\circledR}$ tapes were taken from the left cheek and twenty from the left forearm, respectively. The standardised pressure device was used to apply $225 \mathrm{~g} / \mathrm{cm}^{2}$ of pressure for $5 \mathrm{sec}$. After applying the required pressure the tapes were removed in a single rapid stroke.

\section{IR Densitometry (IRD)}

The reduction in intensity of IR light within a specific diameter of $15 \mathrm{~mm}$ of each removed D-Squame ${ }^{\circledR}$ was quantified using the IR densitometer SquameScan ${ }^{\mathrm{TM}}$ 850A. The density of SC protein content on each removed D-Squame ${ }^{\circledR}$ is thus measured indirectly by spectroscopic absorption of the adhesive tapes. The absorption values (\%) are collected from the display after an equilibration time of 3 min. The linear working range of the instrument as recommended by the manufacturer is between the range of $0 \%$ to $40 \%$ with a resolution of $0.1 \%[24,25]$. Before each set of analysis a clear tape was used as a blank to account for the background noise [24]. The blank was measured and analysed to find the limit of detection (LOD). The LOD for IRD was calculated from the 3.3-fold standard deviation added to the mean background signal of the empty tape. The LOD was calculated using the equation below [38].

Equation 1: $\quad$ LOD $=$ Mean blank $+(3.3 *$ Standard deviation $)$

After absorption measurement by SquameScan ${ }^{\mathrm{TM}} 850 \mathrm{~A}$ the quantity of SC protein adhering on the tape strippings was assayed by the colorimetric $\mu \mathrm{BCA}$ protein assay. 


\section{$\mu \mathrm{BCA}$ Protein Assay}

The quantification of SC protein removed with each tape stripping was carried out according to the microplate working protocol of the $\mu \mathrm{BCA}$ protein assay as previously described [15]. The principle of the $\mu \mathrm{BCA}$ assay is similar to the Lowry procedure. Standard solutions of fresh BGG with a working linear range of 2.5 to $40.0 \mu \mathrm{g} / \mathrm{ml}$ were prepared and used throughout the study [26]. After measurement at $850 \mathrm{~nm}$ each tape strip was placed into a $1.5 \mathrm{ml}$ Eppendorf tube and incubated with $750 \mu \mathrm{l}$ of a $1 \mathrm{M}$ sodium hydroxide solution. The proteins were extracted at $37^{\circ} \mathrm{C}$ and 1,400 rpm for $1 \mathrm{~h}$ inside an orbital shaker. $100 \mu \mathrm{l}$ of the extract were neutralised with an equal volume of $1 \mathrm{M}$ hydrochloric acid. In order to lie within the detectable range of the assay, $100 \mu \mathrm{l}$ of the extract was diluted with $300 \mu \mathrm{l}$ of $1 \mathrm{M}$ sodium chloride solution [26]. Following mixing, $100 \mu$ of each sample was transferred into a 96 well microplate. Subsequently, $100 \mu \mathrm{l}$ of standardized $\mu \mathrm{BCA}$ Protein Assay working reagent was added to each reaction well. The microplate was covered and shaken at $300 \mathrm{rpm}$ for $1 \mathrm{~min}$ and further incubated at $37^{\circ} \mathrm{C}$ for $2 \mathrm{~h}$. The plates were allowed to cool to room temperature and the absorbance of the samples was measured using an UV/VIS microplate reader using a wavelength of $562 \mathrm{~nm}$. The protein content of each tape stripping was analysed in triplicate and the mean value was used for further analysis. Blank D-Squame ${ }^{\circledR}$ tapes were used as controls to minimize background noise. The LOD was calculated using Equation 1 [38]. Calibration curves were prepared with freshly prepared BGG standard solutions for each microplate analysis [15].

\section{Statistical Data Analysis}

A software package SPSS ${ }^{\circledR}$ version $21\left(\right.$ IBM $^{\circledR}$, USA) and Microsoft ${ }^{\circledR}$ Excel 2010 was used to analyse the data. For normally distributed data, parametric statistical tests 
(two way analysis of variance and t-test to compare the means) were used to investigate differences in results for body sites and ethnicities. The non-normally distributed data were analysed using non-parametric tests (Wilcoxon sum of squares). A probability of $p<0.05$ was considered statistically significant. All results are presented as the mean and standard error of the mean (SEM). The correlation coefficient $(R)$ was calculated using Pearson's test. Linear regression was used to determine the coefficients of determination $\left(R^{2}\right)$. Outliers were excluded using interquartile range rule $[39,40]$.

\section{RESULTS}

A total of 60 blank D-Squame® tapes were measured and a similar LOD of $7.21 \%$ and $7.52 \%$ was obtained for both forearm and cheek, respectively [38]. The $\mu \mathrm{BCA}$ assay also showed similar background noise upon comparison of both body sites (Table I). The LOD measurement demonstrated that in both methods the blank values have an effect on the final measured levels.

Table I: The LOD for IRD and $\mu$ BCA assay

\begin{tabular}{|c|c|c|c|c|c|}
\hline & \multicolumn{2}{|c|}{ IRD } & \multicolumn{3}{c|}{$\mu$ BCA assay } \\
\cline { 2 - 6 } & Forearm & Cheek & Forearm & Cheek & BGG \\
\hline LOD & $7.21 \%$ & $7.52 \%$ & $3.65 \mu \mathrm{g}$ & $3.33 \mu \mathrm{g}$ & $0.99 \mu \mathrm{g}$ \\
\hline Tapes below LOD & 0 & 0 & 0 & 0 & 0 \\
\hline
\end{tabular}

The SC protein absorption (\%) using IRD and the protein concentration using the $\mu \mathrm{BCA}$ assay was measured to compare the correlation between two methods. The cumulative SC protein (first nine tape strips) removed from the forearm was higher than the cheek $(p<0.01)$ (Fig.1). 


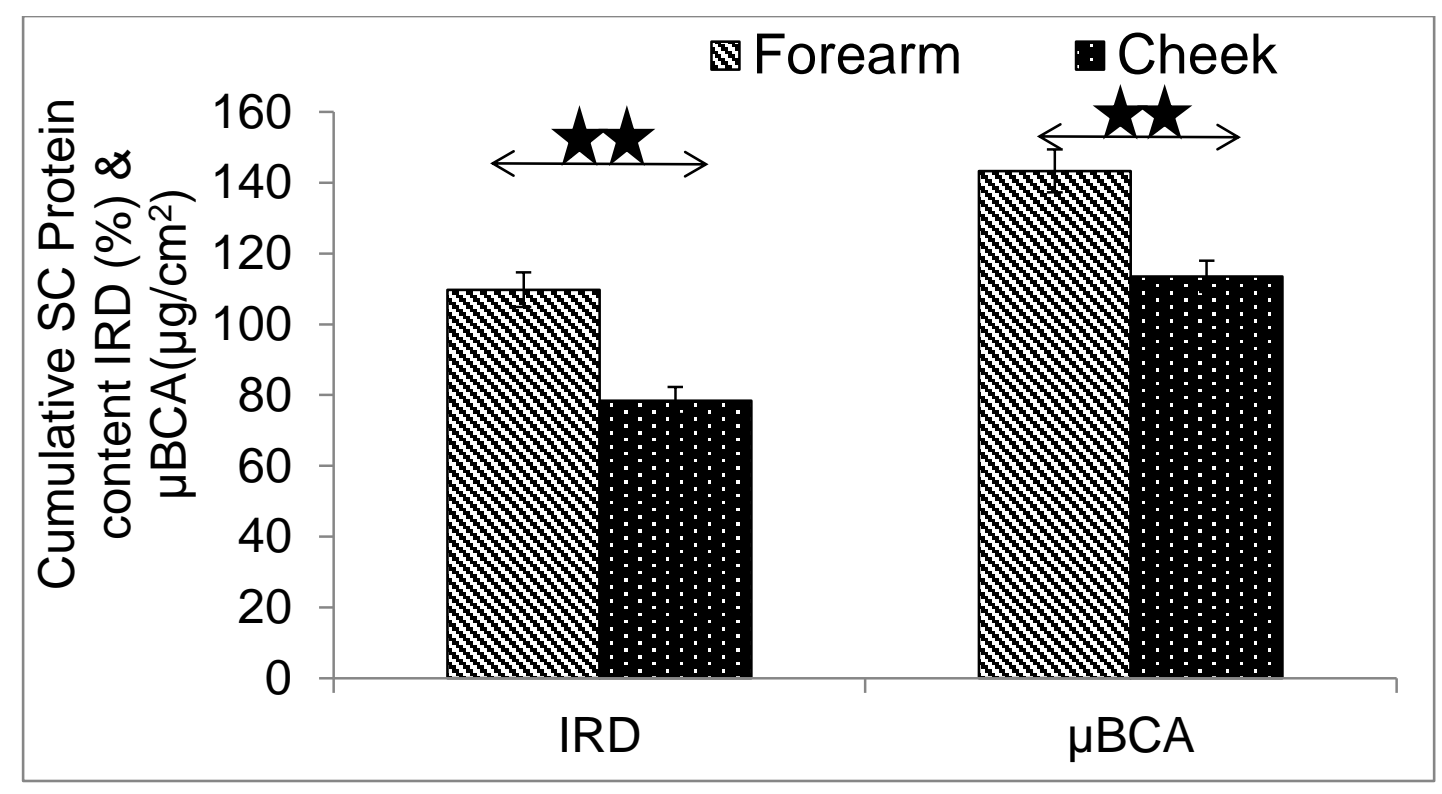

Figure 1: Cumulative SC protein content expressed as average of first nine tapes by IRD and the $\mu \mathrm{BCA}$ protein assay on cheek and forearm of both ethnic groups combined $(n=20$, mean \pm SEM), ${ }^{* *} p<0.01$.

The absolute SC protein levels as assessed by the $\mu \mathrm{BCA}$ assay and IRD followed similar profiles for both Caucasian and Black African subjects. There were no significant differences $(p>0.5)$ between the two ethnicities on each site studied (Fig. 2).

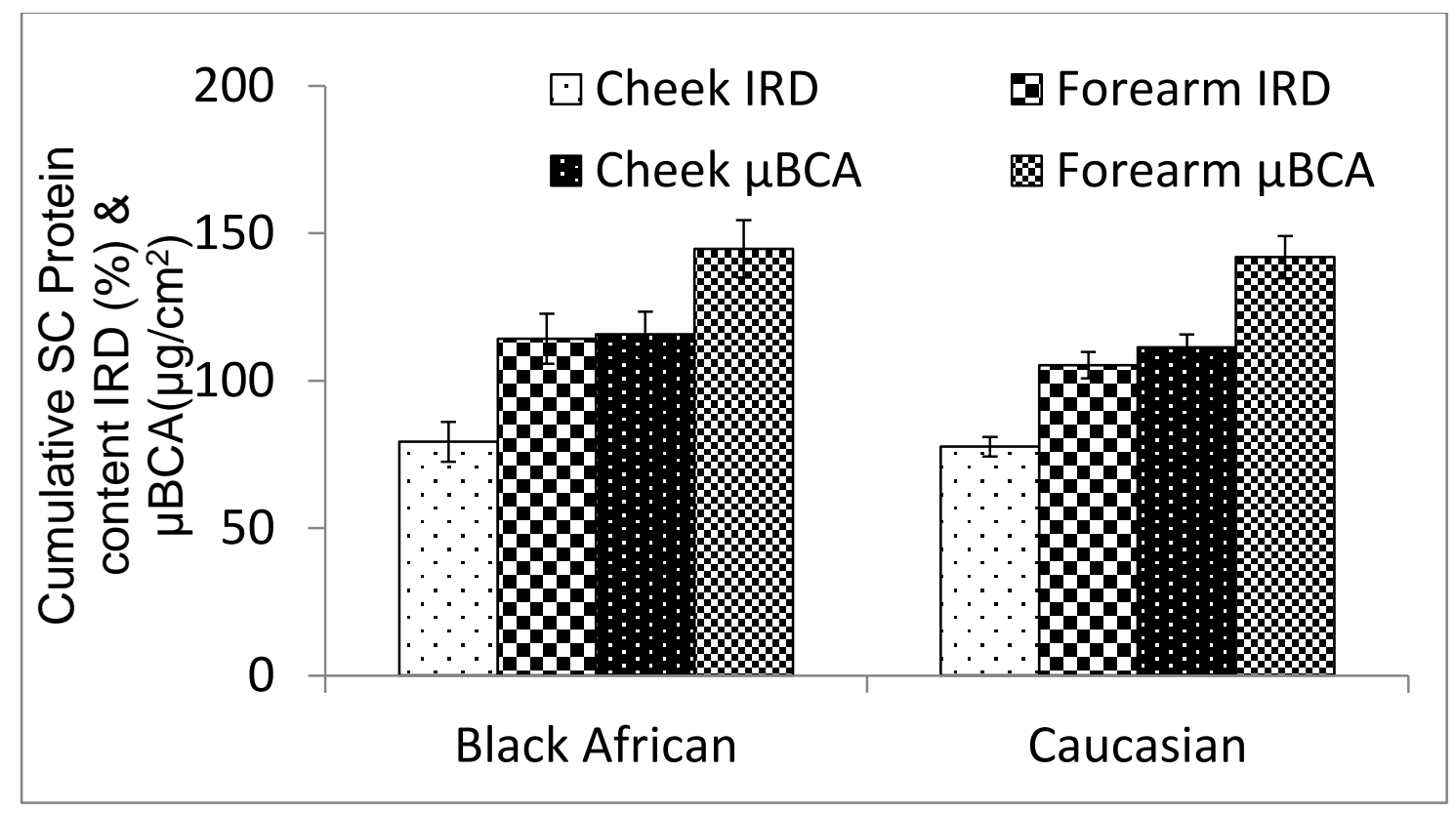

Figure 2: Ethnicity comparison of cumulative SC protein content expressed as average of the first nine tape strippings by IRD and the $\mu \mathrm{BCA}$ protein assay on cheek and forearm $(n=10$ per group, mean \pm SEM) 
In addition, the SC protein content removed decreased with an increase in number of tape strippings in both sites. This trend was observed in IRD and $\mu \mathrm{BCA}$ protein assay (Fig. 3).

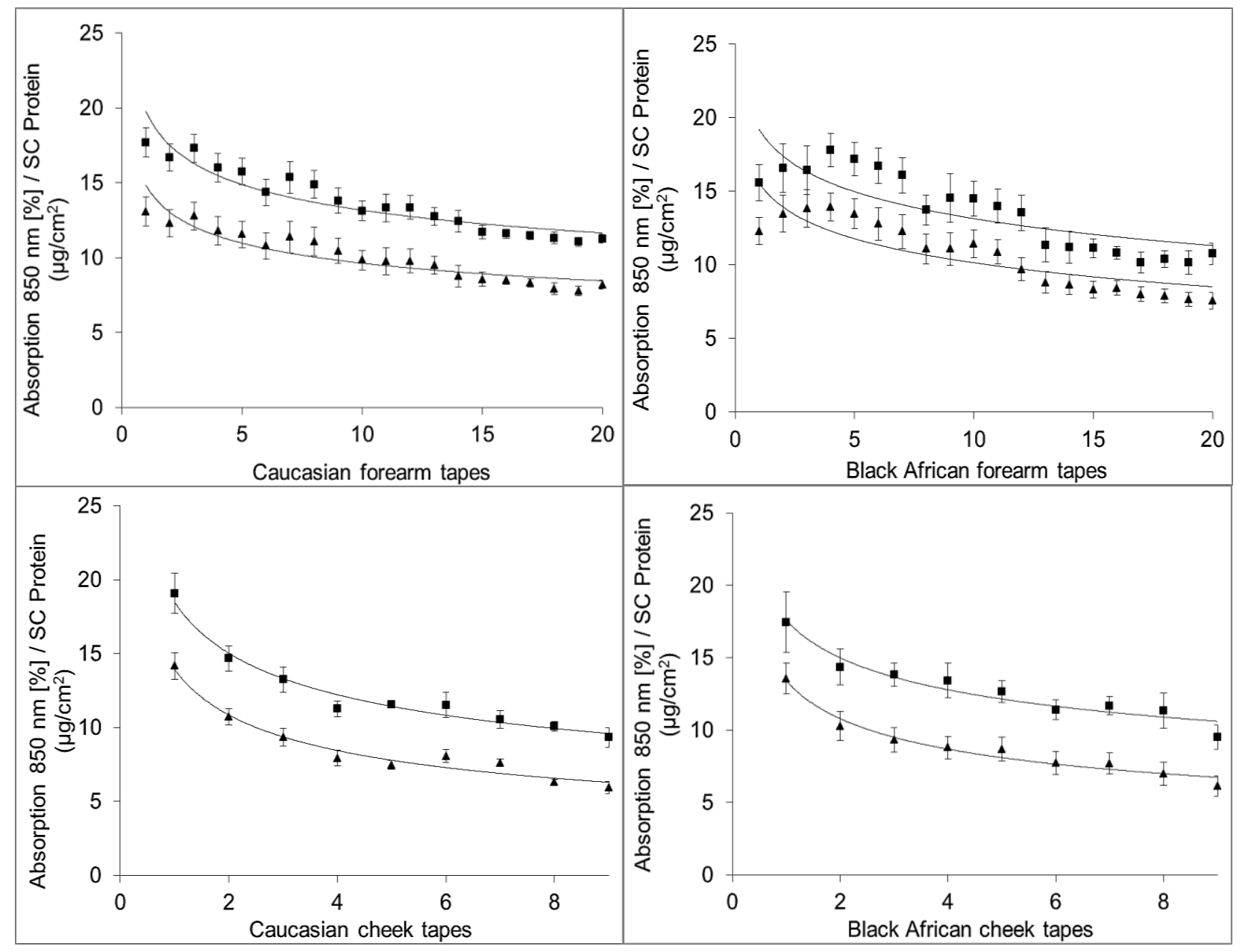

Figure $2: \mu B C A(\square)$ and SquameScan ${ }^{\mathrm{TM}}$ absorption values $(\Delta)$ of 9 sequential tape strippings on cheek and 20 sequential tape strippings on forearm, $(n=10$ per group, mean $\pm S E M)$

The SC protein analysed by both methods from the different sites showed no significant difference $(p>0.05)$ between the Caucasian and Black African subjects. The results showed good correlation between the two methods (Table II). 


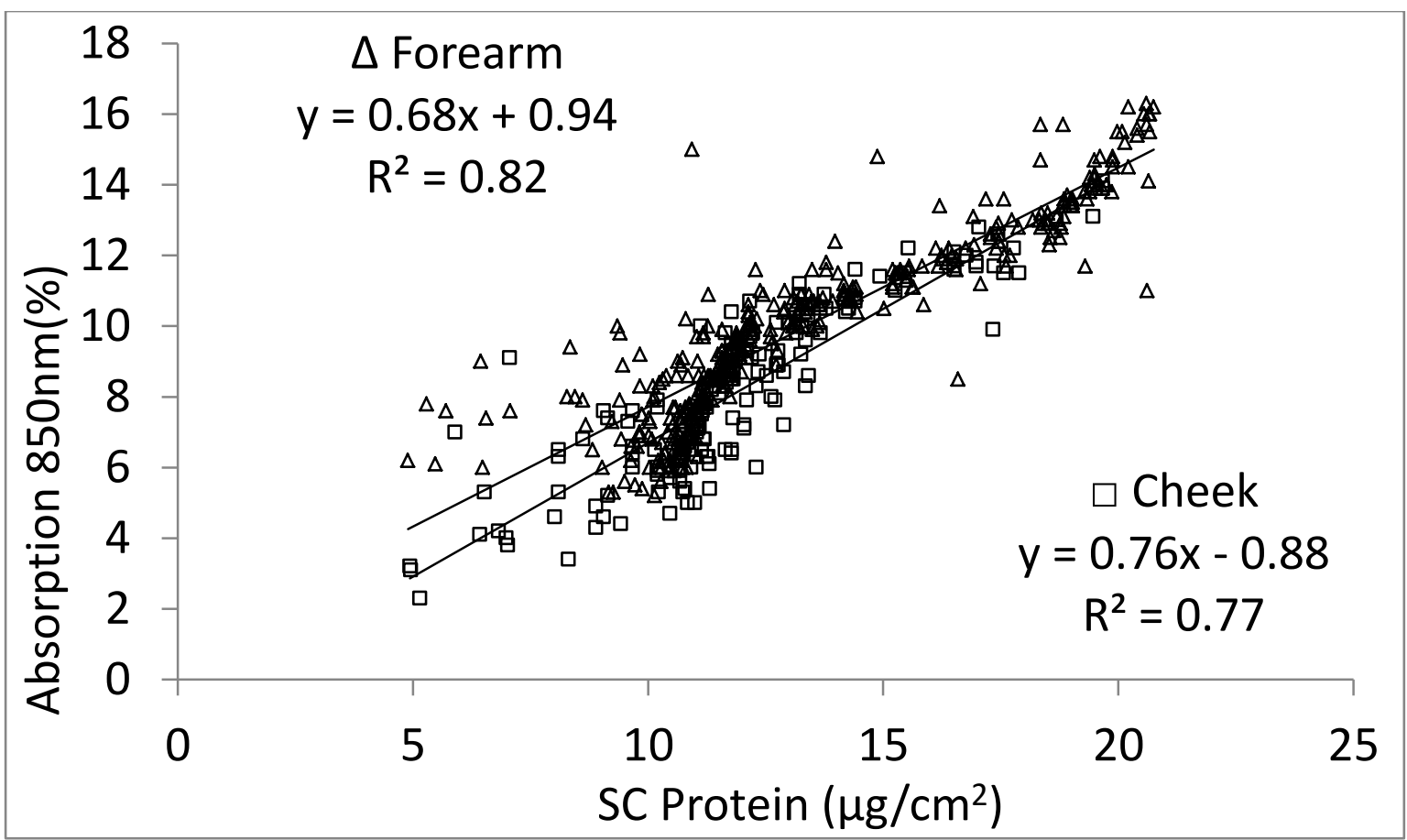

Figure 3: Overall linear regression between $\mu \mathrm{BCA}\left(\mu \mathrm{g} / \mathrm{cm}^{2}\right)$ and SquameScan ${ }^{\mathrm{TM}}$ absorption values $(\%)$ on forearm, $\Delta(20$ tapes, $n=400)$ and cheek, $\square(9$ tapes, $n=$ 180)

The overall coefficient of determination $\left(R^{2}=0.77, n=180\right)$ between IR absorption and colorimetric protein content via the $\mu \mathrm{BCA}$ assay of cheek tape strippings also showed a lower positive linear correlation than the forearm $\left(R^{2}=0.82, n=400\right)($ Fig. 4).

Table II: Overall statistical analysis between the two methods

\begin{tabular}{|c|c|c|c|c|c|}
\hline & Mean & $\mathrm{n}$ & SD & SEM & $\begin{array}{l}\text { Pearson } \\
\text { correlation }\end{array}$ \\
\hline IRD forearm (\%) & 10.35 & 400 & 3.06 & 0.15 & \multirow[t]{2}{*}{$0.91^{* \star}$} \\
\hline$\mu \mathrm{BCA}$ forearm $\left(\mu \mathrm{g} / \mathrm{cm}^{2}\right)$ & 26.13 & 400 & 7.33 & 0.37 & \\
\hline IRD cheek (\%) & 8.72 & 180 & 3.14 & 0.23 & \multirow[t]{2}{*}{$0.85^{\star *}$} \\
\hline$\mu \mathrm{BCA}$ cheek $\left(\mu \mathrm{g} / \mathrm{cm}^{2}\right)$ & 24.05 & 180 & 7.57 & 0.56 & \\
\hline
\end{tabular}


In forearm the Black Africans and Caucasians showed good correlation coefficient between IRD and $\mu B C A$ assay, $0.77(p=0.01)$ and $0.91(p=0.01)$ respectively (Fig. 5).

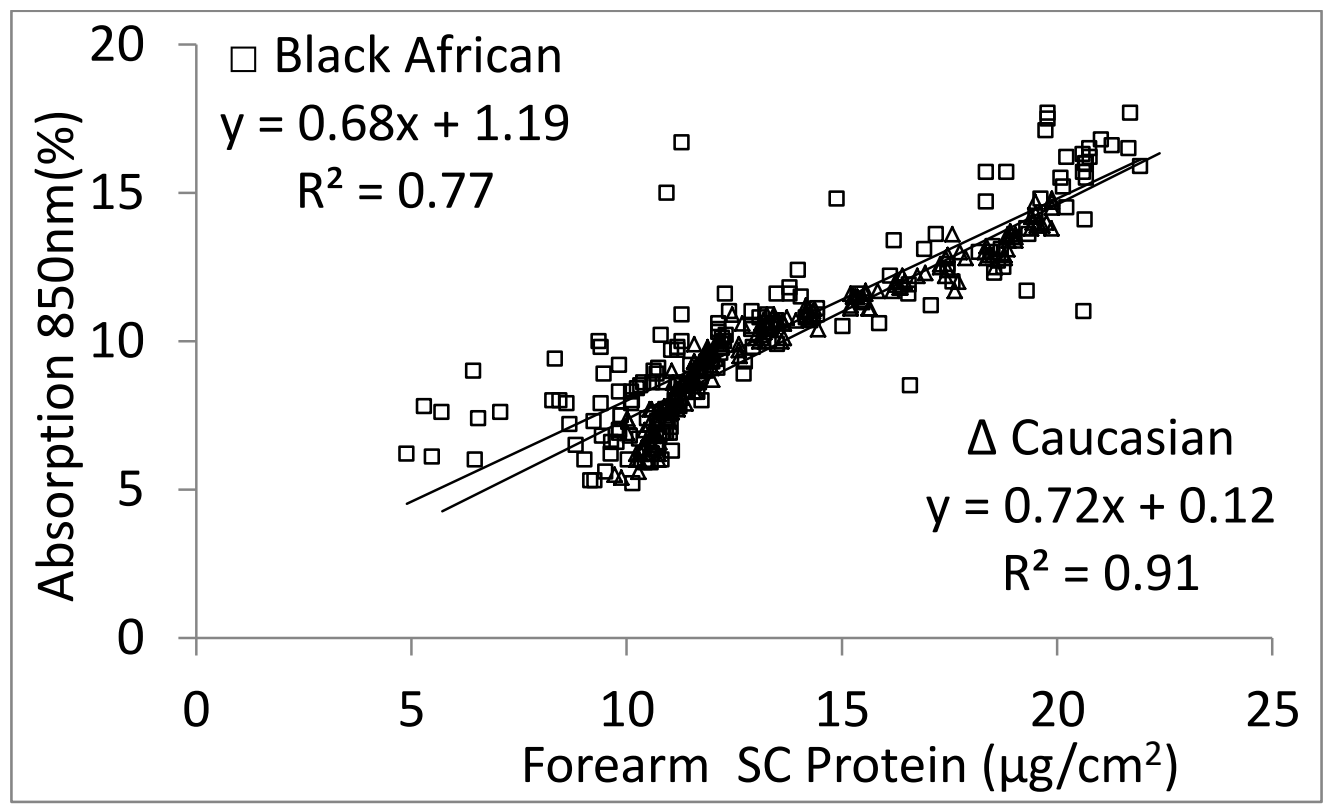

Figure 4: Overall linear regression between $\mu \mathrm{BCA}\left(\mu \mathrm{g} / \mathrm{cm}^{2}\right)$ and SquameScan ${ }^{\mathrm{TM}}$ absorption values (\%) on forearm ( $\mathrm{n}=200$ per group), 10 subjects per group.

For the cheek the Black Africans and Caucasians showed a correlation coefficient of $0.86(p=0.01)$ and $0.69(p=0.01)$ respectively (Fig. 6$)$.

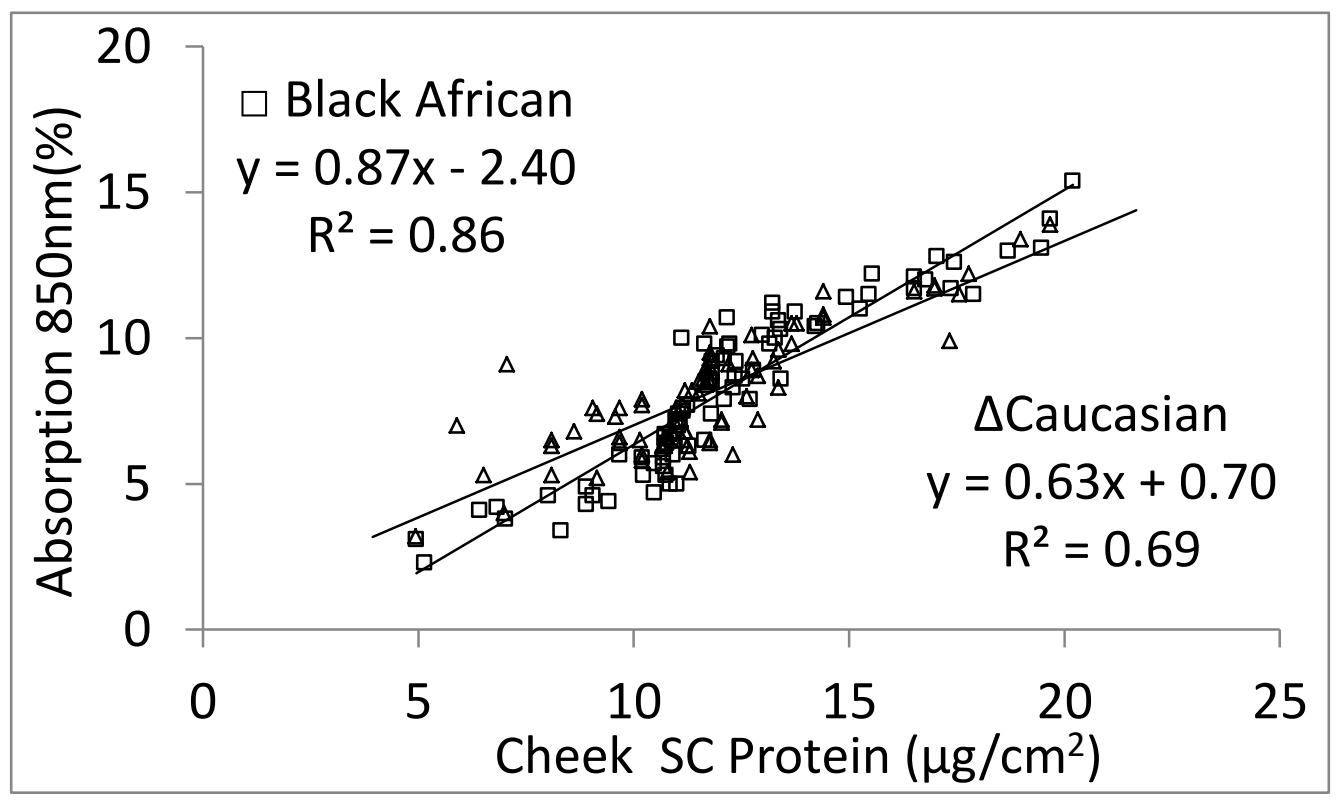

Figure 5: Overall linear regression between $\mu \mathrm{BCA}\left(\mu \mathrm{g} / \mathrm{cm}^{2}\right)$ and SquameScan ${ }^{\mathrm{TM}}$ absorption values (\%) on cheek ( $\mathrm{n}=90$ per group), 10 subjects per group. 


\section{Discussion}

Quantification of SC protein is important from dermatological, pharmaceutical and cosmetic perspectives. Colorimetric methods such as the $\mu \mathrm{BCA}$ assay are well established for SC protein determination following tape stripping of human and porcine SC in vivo and in vitro [24-26]. In this study we compared the $\mu \mathrm{BCA}$ assay and IRD for SC protein profiling of two body sites (cheek and forearm) and of two ethnicities (Caucasian and Black African females).

The correlation coefficients were similar to published data for forearm and shoulder tape strippings of Caucasian subjects [24]. In our study the $\mu \mathrm{BCA}$ assay was more sensitive. The increased sensitivity was observed for both anatomical sites and the two ethnic groups. The $\mu \mathrm{BCA}$ assay extracts the whole tape and analyses the precise colorimetric difference in a controlled environment. On the other hand, the IRD suffers from loss of absorption of reflected rays due to scattering and other physical factors. Nevertheless, the loss of absorption is constant; hence the ratio of the total SC protein measurement is consistent even though the values are slightly lower than that of colorimetric assay.

The amount of protein removed by each D-Squame ${ }^{\circledR}$ for each subject showed a decrease in SC protein with increasing tape number. The initial tape strippings showed higher values as these corneocytes are less cohesive and adhere to the tapes more easily than corneocytes from deeper SC layers. As the tape strip number increased the corneocyte cohesion also increased; hence there was a decrease in amount of protein recovered from tape strippings. A transient drop in protein recovery was observed during sequential tape stripping of forearm (tapes 6-8) in both methods (Fig. 3). The change of transitional layers between stratum disjunctum 
and stratum compactum may be related to the drop in the protein recovered. This may also be attributed to the level of hydration leading to a lower SC protein removal [41]. This non-uniform SC removal by consecutive tape stripping was evident for both body sites investigated. Our findings are in line with previous studies which observed non-uniform SC recovery from sequential tape stripping [24-26, 42]. In addition, increased skin hydration probably contributes to the decreased adhesiveness to the tapes in deeper layers of SC. Water content within the SC layers increases as a function of depth which can lead to swollen and strong corneocytes $[43,44]$ making them less adherent to tapes.

Cheek and forearm showed statistically significant differences in quantity of SC harvested on sequential tape strip strippings, which is in line with previous findings [30]. Cheek showed lower cumulative protein content in $\mu \mathrm{BCA}$ assay $\left(113 \mu \mathrm{g} / \mathrm{cm}^{2} \pm\right.$ 4.4) and lower cumulative absorption value in IRD $(78.5 \% \pm 3.8)$. The calculated values for cheek showed a percentage difference of $26 \%$ (lower) than forearm $\mu \mathrm{BCA}$ assay $\left(143 \mu \mathrm{g} / \mathrm{cm}^{2} \pm 6.1\right)$ and an average of $39 \%$ lower values for IRD readings (110 $\% \pm 4.9)$. The finding that higher amounts of SC protein were removed from the forearm than the cheek is in contrast to previous literature, where cheek recorded significantly higher total SC protein than forearm [45]. The previous reports by Yamaguchi et al. suggests that the total protein from cheek areas was higher than that of mid-ventral forearm because of increased desquamation due to sun exposure in cheeks [45]. The difference in ethnicity (Japanese Asians), age differences and the environmental factors may be considered as factors for the difference in SC properties [46]. Nevertheless, our findings of lower cumulative SC protein recovery from cheeks are consistent with Voegeli et al. [30]. 
It was evident that the initial tape strippings removed higher amounts of SC protein in most of the subjects. The increased protein content in early tapes could be due to loosely bound outer corneocytes and the values progressively decreased as the tape number increased [20]. Nevertheless, we found a high correlation between IR absorption values and SC protein values for both anatomical sites studied. This is in line with the findings of Mohammed et al. and Voegeli et al. [24, 47]. We found a good linear correlation $\left(R^{2}=0.82, n=400\right)$ between IR absorption of forearm tape strippings and mass of SC protein using the colorimetric method (Fig. 4). This concurs with the findings of Voegeli et al. and Hahn et al. who reported a linear correlation of $R^{2}=0.85$ and $R^{2}=0.63$, respectively. The IRD and $\mu B C A$ assays showed a similar pattern in SC protein recovery from tape strippings collected from the subjects of both ethnicities. There was no significant difference $(p>0.05)$ between Black African and Caucasian subjects for total SC protein recovered.

Even though there is a good correlation between the two methods, the BCA assay provides the absolute values, whereas IRD uses an approximation. The $\mu \mathrm{BCA}$ assay analyses the whole tape strip, whereas IRD passes a beam of light (wavelength 850 $\mathrm{nm}$ ) through a central area of $15 \mathrm{~mm}$ diameter and therefore does not scan the entire tape, as the IR beam only passes through the window covering $45 \%$ of the tape area [26]. From the LOD measurements, there is a need to deducting the background noise to determine the SC protein levels in both methods. Taking into account the non-destructive approach and relatively quicker analysis time the IRD method is a superior method. 


\section{Conclusion}

This study is the first report to compare IRD with the $\mu \mathrm{BCA}$ assay for profiling SC protein on facial and forearm tape strippings for Caucasian and Black African subjects. We found good correlation between the two methods in both body sites studied. This study was necessary, as facial SC is morphologically and functionally distinct from forearm or shoulder SC and the method had not been investigated for different ethnic groups previously. The non-destructive method of SC protein quantification, underpins the ongoing studies to quantify biomarkers of facial skin on different ethnic groups.

The resultant calibration curves may be used as a rapid indirect protein assessment of tape strippings from the cheek area to reduce the errors by relying on optical method alone. The study adds further support for the application of IRD, as a fast and non-destructive method for quantification of SC protein on tape strippings. To date IRD has been shown to be suitable for in vivo and in vitro analysis of porcine and human SC (Caucasians and Black Africans). Other body sites, ethnicities, age groups and groups with different skin conditions should be investigated in the future.

\section{Acknowledgements}

The Authors wish to thank the Photobiology Laboratory, Dept of Pharmacy, Sefako Mkgatho University (previously Medunsa Campus, UL) South Africa for the samples. This work was financial supported by DSM Nutritional Products Ltd., Basel, Switzerland and UCL overseas research scholarship, 2011-2015. 


\section{References}

1. Matts, P.J. Water, water everywhere...? Inter J Cosmetic Sci. 31(5):402 (2009).

2. Hadgraft, J. and Lane, M.E. Transepidermal water loss and skin site: a hypothesis. Int J Pharm. 373(1):1-3 (2009).

3. Machado, M., Salgado, T.M., Hadgraft, J. and Lane, M.E. The relationship between transepidermal water loss and skin permeability. Int $J$ Pharm. 384(1):73-7 (2010).

4. Holbrook, K.A. and Odland, G.F. Regional differences in the thickness (cell layers) of the human stratum corneum: an ultrastructural analysis. J Invest Dermatol. 62(4):415-22 (1974).

5. Fluhr, J.W., Kao, J., Jain, M., Ahn, S.K., Feingold, K.R. and Elias, P.M. Generation of free fatty acids from phospholipids regulates stratum corneum acidification and integrity. J Invest Dermatol. 117(1):44-51 (2001).

6. Kao, J.S., Fluhr, J.W., Man, M.-Q., Fowler, A.J., Hachem, J.-P., Crumrine, D., Short-term glucocorticoid treatment compromises both permeability barrier homeostasis and stratum corneum integrity: inhibition of epidermal lipid synthesis accounts for functional abnormalities. J Invest Dermatol. 120(3):456-64 (2003).

7. Bornkessel, A., Flach, M., Arens-Corell, M., Elsner, P. and Fluhr, J. Functional assessment of a washing emulsion for sensitive skin: Mild impairment of stratum corneum hydration, ph, barrier function, lipid content, integrity and cohesion in a controlled washing test. Skin Res Technol. 11 (1):53-60 (2005). 
8. Kalia, Y.N., Pirot, F. and Guy, R.H. Homogeneous transport in a heterogeneous membrane: water diffusion across human stratum corneum in vivo. Biophys J. 71(5):2692 (1996).

9. Löffler, H., Dreher, F. and Maibach, H. Stratum corneum adhesive tape stripping: influence of anatomical site, application pressure, duration and removal. $\mathrm{Br}$ J Dermatol. 151(4):746-52 (2004).

10. Pinkus, $H$. Examination of the epidermis by the strip method of removing horny layers. J Invest Dermatol. 16:383-6 (1951).

11. Pinkus, H. Examination of the epidermis by the strip method. $J$ Invest Dermatol. 19(6):431-47 (1952).

12. Lademann, J., Meinke, M., Schanzer, S., Richter, H., Darvin, M., Haag, S., In vivo methods for the analysis of the penetration of topically applied substances in and through the skin barrier. Inter J Cosmetic Sci. 34(6):551-9 (2012).

13. Bashir, S.J., Chew, A.L., Anigbogu, A., Dreher, F. and Maibach, H.I. Physical and physiological effects of stratum corneum tape stripping. Skin Res Technol. $7(1): 40-8$ (2001)

14. Marttin, E., Neelissen-Subnel, M., De Haan, F. and Bodde, H. A critical comparison of methods to quantify stratum corneum removed by tape stripping. Skin Pharmacol Physiol. 9(1):69-77 (1996).

15. Myer, K. and Maibach, H. Stratum corneum evaluation methods: overview. Skin Res Technol. 19(3):213-9 (2013). 
16. Jacobi, U., Meykadeh, N., Sterry, W. and Lademann, J. Effect of the vehicle on the amount of stratum corneum removed by tape stripping. JDDG: Journal Deut Dermatol Gesel. 1(11):884-9 (2003).

17. Breternitz, M., Flach, M., Prassler, J., Elsner, P. and Fluhr, J.W. Acute barrier disruption by adhesive tapes is influenced by pressure, time and anatomical location: integrity and cohesion assessed by sequential tape stripping. A randomized, controlled study. Br J Dermatol. 156(2):231-40 (2007).

18. Van der Molen, R., Spies, F.t., Van't Noordende, J., Boelsma, E., Mommaas, A. and Koerten, H. Tape stripping of human stratum corneum yields cell layers that originate from various depths because of furrows in the skin. Arch Dermatol Res. 289(9):514-8 (1997).

19. Diffey, B. A mathematical model for ultraviolet optics in skin. Phys Med Biol. 28(6):647 (1983).

20. Dreher, F., Arens, A., Hostynek, J., Mudumba, S., Ademola, J. and Maibach, H. Colorimetric method for quantifying human stratum corneum removed by adhesive-tape-stripping. Acta Derm Venereol (Oslo). 78:186-9 (1998).

21. Dreher, F., Modjtahedi, B., Modjtahedi, S. and Maibach, H. Quantification of stratum corneum removal by adhesive tape stripping by total protein assay in 96 -well microplates. Skin Res Technol. 11(2):97-101 (2005).

22. Weigmann, H.-J., Lademann, J., Meffert, H., Schaefer, H. and Sterry, W. Determination of the horny layer profile by tape stripping in combination with optical spectroscopy in the visible range as a prerequisite to quantify percutaneous absorption. Skin Pharmacol Physiol. 12(1-2):34-45 (1999). 
23. Lindemann, U., Wilken, K., Schaefer, H. and Sterry, W. Quantification of the horny layer using tape stripping and microscopic techniques. $J$ Biomed Opt. 8(4):601-7 (2003).

24. Voegeli, R., Heiland, J., Doppler, S., Rawlings, A. and Schreier, T. Efficient and simple quantification of stratum corneum proteins on tape strippings by infrared densitometry. Skin Res Technol. 13(3):242-51 (2007).

25. Hahn, T., Hansen, S., Neumann, D., Kostka, K.-H., Lehr, C.-M., Muys, L., Infrared densitometry: a fast and non-destructive method for exact stratum corneum depth calculation for in vitro tape-stripping. Skin Pharmacol Physiol. 23(4):183-92 (2010).

26. Klang, V., Schwarz, J., Hartl, A. and Valenta, C. Facilitating in vitro tape stripping: application of infrared densitometry for quantification of porcine stratum corneum proteins. Skin Pharmacol Physiol. 24(5):256-68 (2011).

27. Mohammed, D., Yang, Q., Guy, R., Matts, P., Hadgraft, J. and Lane, M. Comparison of gravimetric and spectroscopic approaches to quantify stratum corneum removed by tape-stripping. Eur J Pharm Biopharm. 82(1):171-4 (2012).

28. Weigand, D.A., Haygood, C. and Gaylor, J.R. Cell layer and density of Negro and Caucasian stratum corneum. J Invest Dermatol. 62(6):563-8 (1974).

29. Rawlings, A. Molecular basis for stratum corneum maturation and moisturization. Br J Dermatol. 171(s3):19-28 (2014).

30. Voegeli, R., Rawlings, A., Doppler, S., Heiland, J. and Schreier, T. Profiling of serine protease activities in human stratum corneum and detection of a stratum corneum tryptase-like enzyme. Inter J Cosmetic Sci. 29 (3):191-200 (2007). 
31. Plewig, G. and Jansen, T. Size and shape of corneocytes: Variation with anatomic site and age; in Wilhelm, K P., Berardesca, E., Elsner, P., Maibach, H I., (eds): Bioengineering of the Skin: Skin Surface Imaging and Analysis. CRC press, Boca Raton. 181-96 (1997).

32. Egawa, M., Hirao, T. and Takahashi, M. In vivo estimation of stratum corneum thickness from water concentration profiles obtained with Raman spectroscopy. Acta Derm Venereol (Oslo). 87(1):4-8 (2007).

33. Ya-Xian, Z., Suetake, T. and Tagami, H. Number of cell layers of the stratum corneum in normal skin-relationship to the anatomical location on the body, age, sex and physical parameters. Arch Dermatol Res. 291(10):555-9 (1999).

34. Bowser, P. and White, R. Isolation, barrier properties and lipid analysis of stratum compactum, a discrete region of the stratum corneum. $\mathrm{Br} J$ Dermatol. 112(1):1-14 (1985).

35. Voegeli, R., Rawlings, A., Doppler, S. and Schreier, T. Increased basal transepidermal water loss leads to elevation of some but not all stratum corneum serine proteases. Inter J Cosmetic Sci. 30(6):435-42 (2008).

36. Rawlings, A.V. and Voegeli, R. Stratum corneum proteases and dry skin conditions. Cell Tissue Res. 351(2):217-35 (2013).

37. Fluhr, J.W., Kao, J., Jain, M., Ahn, S.K., Feingold, K.R. and Elias, P.M. Generation of free fatty acids from phospholipids regulates stratum corneum acidification and integrity. J Invest Dermatol. 117(1):44-51 (2001).

38. Little, T.A. Method Validation Essentials, Limit of Blank, Limit of Detection, and Limit of Quantitation., Bio Pharm International, 28(4): 48-51 (2015). 
39. Ghasemi, A. and Zahediasl, S. Normality tests for statistical analysis: A guide for non-statisticians. Int J Endocrinol Metabol. 10(2):486 (2012).

40. Peat, J. and Barton, B. Medical statistics: A guide to data analysis and critical appraisal: John Wiley \& Sons (2008).

41. Feng, L., Chandar, P., Lu, N., Vincent, C., Bajor, J. and McGuiness, H. Characteristic differences in barrier and hygroscopic properties between normal and cosmetic dry skin. II. Depth profile of natural moisturizing factor and cohesivity. Inter J Cosmetic Sci. 36(3):231-8 (2014).

42. Lu, N., Chandar, P., Tempesta, D., Vincent, C., Bajor, J. and McGuiness, H. Characteristic differences in barrier and hygroscopic properties between normal and cosmetic dry skin. I. Enhanced barrier analysis with sequential tape stripping Inter $J$ Cosmetic Sci. 36(2):167-74 (2014).

43. Boncheva, M., De Sterke, J., Caspers, P.J. and Puppels, G.J. Depth profiling of Stratum corneum hydration in vivo: a comparison between conductance and confocal Raman spectroscopic measurements. Exp Dermatol. 18(10):870-6 (2009).

44. Crowther, J., Sieg, A., Blenkiron, P., Marcott, C., Matts, P., Kaczvinsky, J., Measuring the effects of topical moisturizers on changes in stratum corneum thickness, water gradients and hydration in vivo. $\mathrm{Br} J$ Dermatol. 159(3):567-77 (2008).

45. Yamaguchi, M., Tahara, Y., Makino, T. and Shimizu, T. Comparison of cathepsin L activity in cheek and forearm stratum corneum in young female adults. Skin Res Technol. 15(3):370-5 (2009). 
46. Rawlings, A.V. Ethnic skin types: are there differences in skin structure and function?. Int J Cosmet Sci. 28(2):79-93 (2006).

47. Mohammed, D., Matts, P.J., Hadgraft, J. and Lane, M.E. Variation of stratum corneum biophysical and molecular properties with anatomic site. AAPS J. 14(4):806-12 (2012). 